\title{
Pseudoaneurisma traumático da artéria meníngea média tratado por via endovascular
}

\author{
Daniel Gomes Gonçalves Neto', Guilherme Brasileiro de Aguiar', \\ José Carlos Esteves Veiga ${ }^{3}$, Márcio Alexandre Teixeira da Costa 4 , \\ Maurício Jory², Nelson Saade ${ }^{5}$, Mário Luiz Marques Conti ${ }^{6}$
}

Disciplina de Neurocirurgia, Departamento de Cirurgia, Faculdade de Ciências Médicas da Santa Casa de Misericórdia de São Paulo, São Paulo, SP, Brasil.

\section{RESUMO}

Os pseudoaneurismas traumáticos da artéria meníngea média (AMM) representam lesões raras, correspondendo a menos de 1\% dos aneurismas intracranianos. Em geral, estão associados à fratura craniana temporal que cruza o trajeto da AMM. O hematoma extradural (HED) é a apresentação mais comum desse tipo de lesão, podendo apresentar elevada morbimortalidade na maioria dos casos. $O$ diagnóstico dos pseudoaneurismas da AMM pode ser realizado por angiorressonância, angiotomografia e, principalmente, por arteriografia cerebral. Após a confirmação de sua existência, o tratamento é mandatório e deve ser realizado precocemente, por causa do risco potencial de ruptura. Esse tratamento pode ser realizado por craniotomia e coagulação da artéria meníngea média, ou por via endovascular com oclusão do aneurisma. Apresentamos neste relato o caso de paciente vítima de traumatismo craniano atendido em nosso serviço. Os exames de imagem iniciais mostravam fratura temporal, associada à contusão hemorrágica adjacente. O paciente foi submetido à angiografia cerebral, sendo diagnosticado um pseudoaneurisma na artéria meníngea média. Ele foi submetido a procedimento endovascular para embolização do aneurisma, tendo evoluído satisfatoriamente.

\section{PALAVRAS-CHAVE}

Traumatismos craniocerebrais, artérias meníngeas, falso aneurisma, traumatismo cerebrovascular; embolização terapêutica.

\section{ABSTRACT}

Traumatic pseudoaneurysm of the middle meningeal artery treated by endovascular intervention

The traumatic pseudoaneurysms of the middle meningeal artery (MMA) are rare lesions, accounting for less than $1 \%$ of all intracranial aneurysms. They are associated mainly to temporal skull fracture that crosses the path of MMA. The epidural hematoma is the most common presentation of this type of injury, and may have high morbidity and mortality in most cases. The diagnosis of pseudoaneurysm of the MMA can be performed by MRI-angiography, CT-angiography, and mainly by digital cerebral arteriography. After confirming its existence, treatment is mandatory and should be performed early, due to the potential risk of rupture. This treatment can be performed by craniotomy and coagulation of the middle meningeal artery, or by endovascular intervention, with occlusion of the aneurysm. We present here the case of a patient with a head trauma, who was admitted to our service. The initial CT imaging demonstrated a temporal fracture, associated with hemorrhagic contusion adjacent. The patient underwent cerebral angiography, being diagnosed with a middle meningeal artery aneurysm. He was submitted to an endovascular embolization of the aneurysm, having evolved satisfactorily.

\section{KEYWORDS}

Craniocerebral trauma, meningeal arteries, pseudoaneurysm, cerebrovascular trauma, embolization therapeutic.

1 Neurocirurgião, estagiário de Neurocirurgia Endovascular, Serviço de Neurocirurgia, Irmandade da Santa Casa de Misericórdia de São Paulo (ISCMSP), São Paulo, SP, Brasil.

2 Neurocirurgião e neurorradiologista intervencionista, Serviço de Neurocirurgia, ISCMSP, São Paulo, SP, Brasil.

3 Neurocirurgião, chefe do Serviço de Neurocirurgia, ISCMSP, professor adjunto livre-docente da Faculdade de Ciências Médicas da Santa Casa de São Paulo (FCMSCSP), São Paulo, SP, Brasil.

4 Residente de Neurocirurgia, Serviço de Neurocirurgia, ISCMSP, São Paulo, SP, Brasil.

5 Neurocirurgião, Serviço de Neurocirurgia, ISCMSP, professor instrutor da FCMSCSP, São Paulo, SP, Brasil.

6 Neurocirurgião e neurorradiologista intervencionista, Serviço de Neurocirurgia, ISCMSP, professor-assistente doutor da FCMSCSP, São Paulo, SP, Brasil. 


\section{Introdução}

O pseudoaneurisma pós-traumático da artéria meníngea média (AMM) representa lesão rara, ${ }^{1-6}$ correspondendo a menos de $1 \%$ dos aneurismas intracranianos. ${ }^{2,3}$ Tais lesões estão associadas principalmente a fraturas cranianas traumáticas, ${ }^{7-9}$ possuindo altas taxas de mortalidade. . $^{1,2,4,6} \mathrm{O}$ hematoma extradural é a apresentação precoce ou tardia mais comum desse tipo de lesão, ${ }^{8}$ podendo estar associado também a hematoma subdural ou hemorragia subaracnóidea. ${ }^{2,3}$

O objetivo do presente artigo é apresentar o caso de um paciente vítima de traumatismo craniano que desenvolveu pseudoaneurisma da AMM, sendo submetido a tratamento endovascular com sucesso. Realizou-se, ainda, breve revisão da literatura sobre o tema.

\section{Relato de caso}

Paciente de 52 anos de idade, masculino, foi admitido em nosso serviço após sofrer traumatismo cranioencefálico (TCE) por queda de telhado. Ao exame neurológico inicial, apresentava-se alerta, porém desorientado e com períodos de agitação psicomotora. Pontuação 14 na escala de coma de Glasgow. Não tinha défices motores ou de nervos cranianos.

Foi submetido à tomografia computadorizada (TC) de crânio que evidenciou fratura temporal direita, contusão hemorrágica adjacente e hemorragia subaracnóidea, além de fratura em base de crânio, adjacente ao canal carotídeo (Figura 1). Por causa da possibilidade de lesão da artéria carótida interna
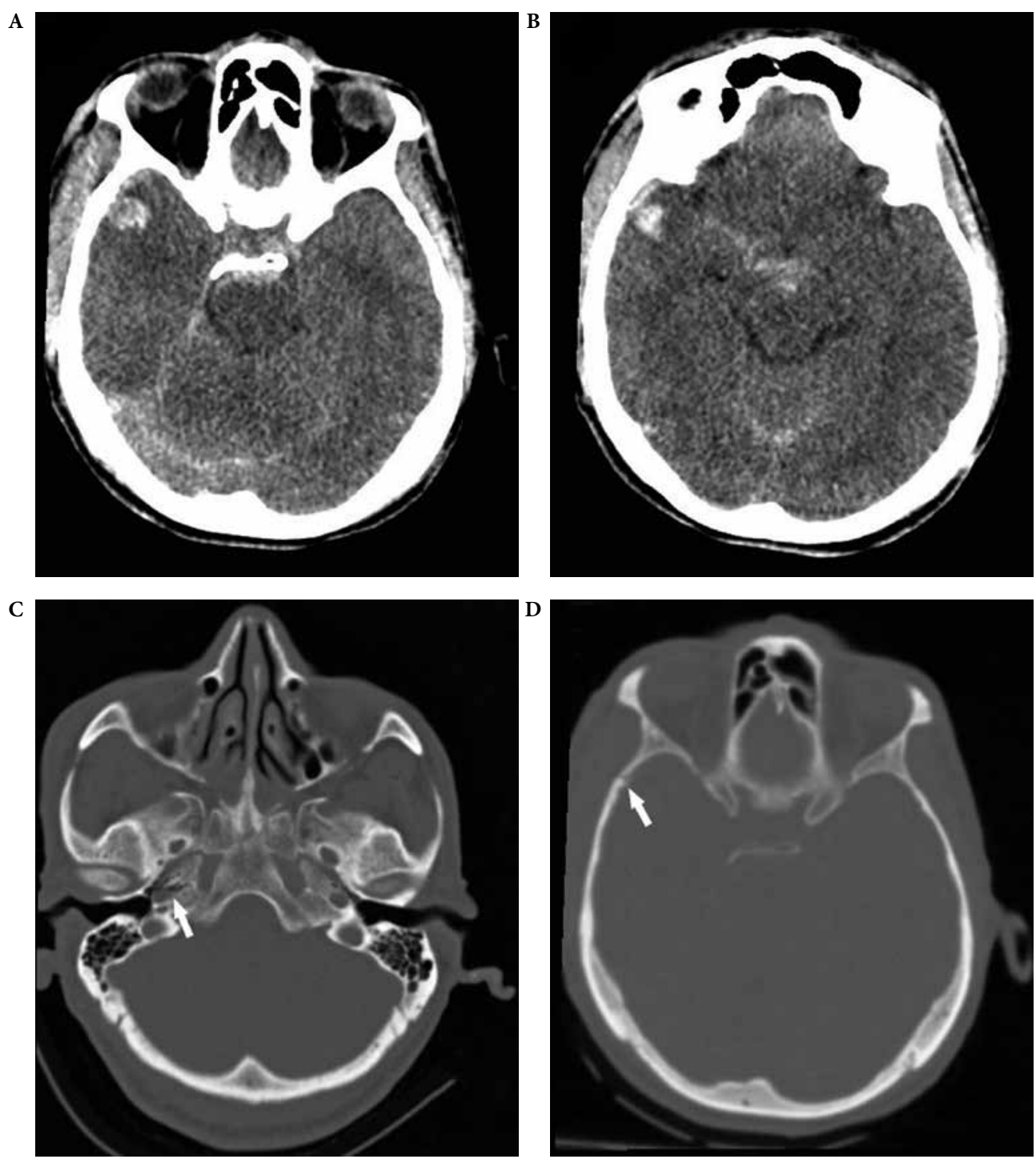

Figura 1 - Tomografia computadorizada de crânio (axial) demonstrando: (A-B) contusão hemorrágica temporal direita, associada à hemorragia subaracnóidea na fissura sylviana direita e nas cisternas da base; (C) fratura na base do crânio, adjacente ao canal carotídeo direito (seta); (D) fratura temporal à direita (seta). 
(ACI) em decorrência da fratura do canal carotídeo, foi realizada angiografia cerebral (AGC). A AGC não demonstrou lesão da ACI, porém mostrou pseudoaneurisma na artéria meníngea média direita, adjacente à fratura temporal (Figura 2).
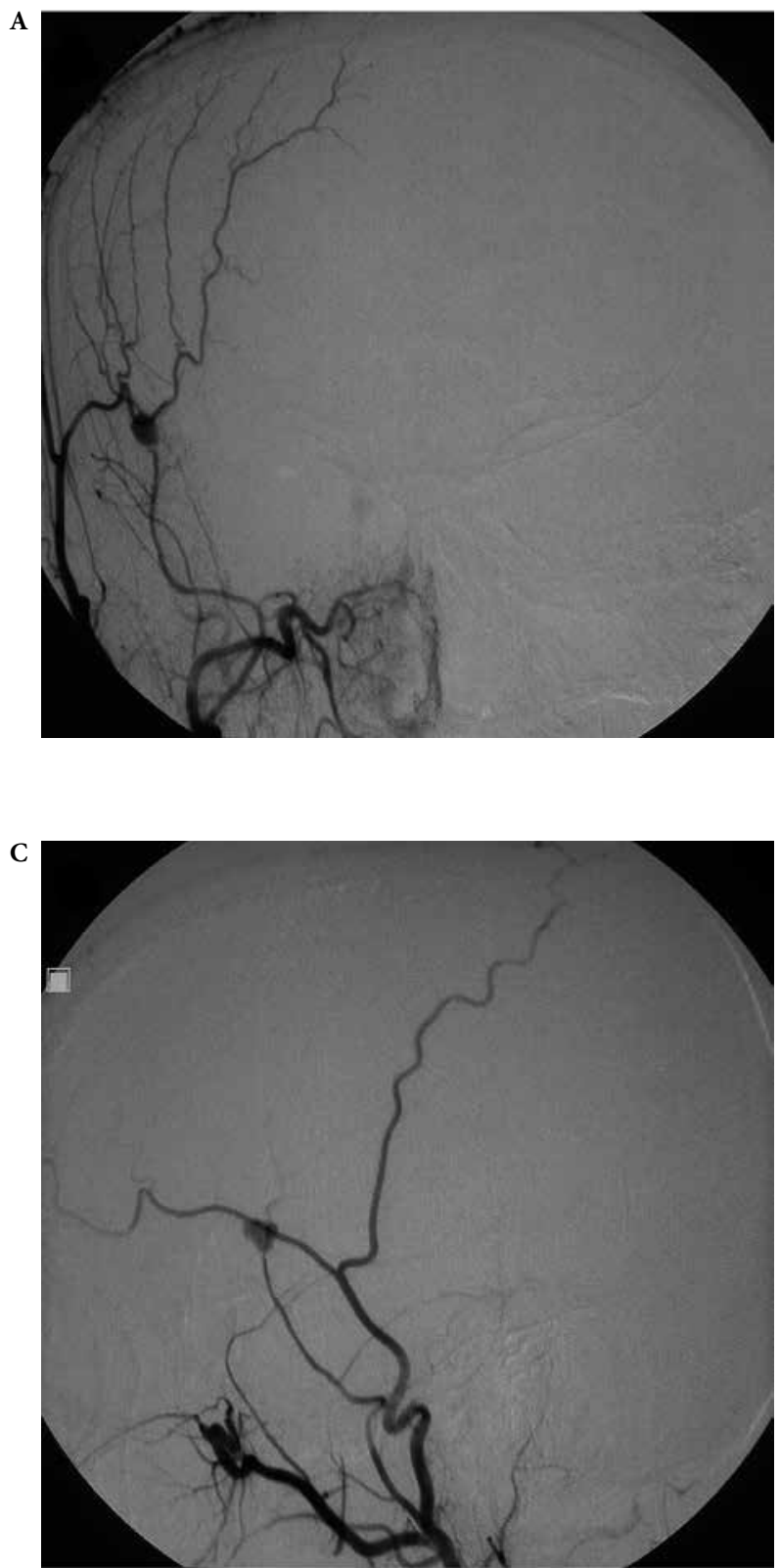

B

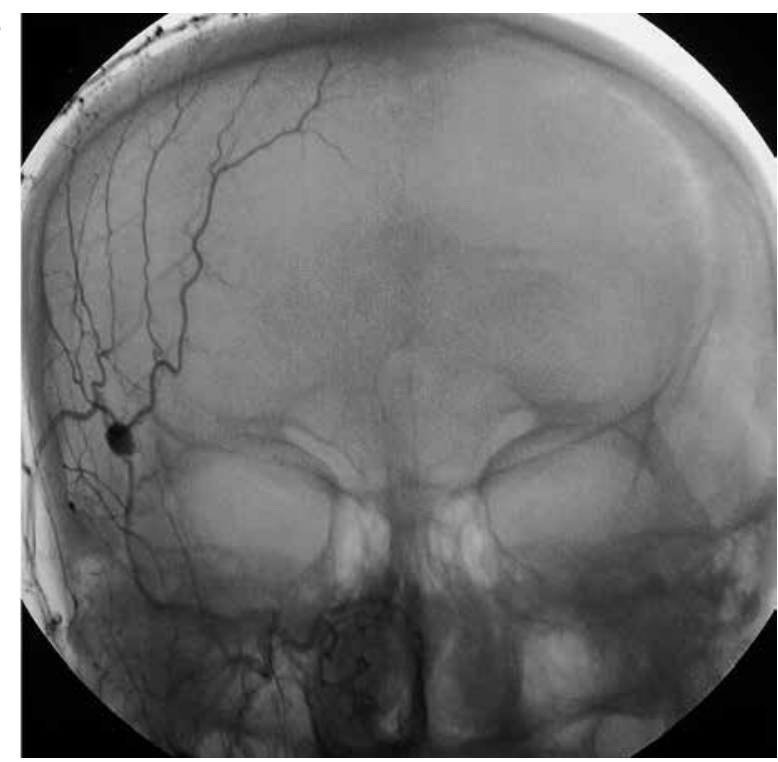

D

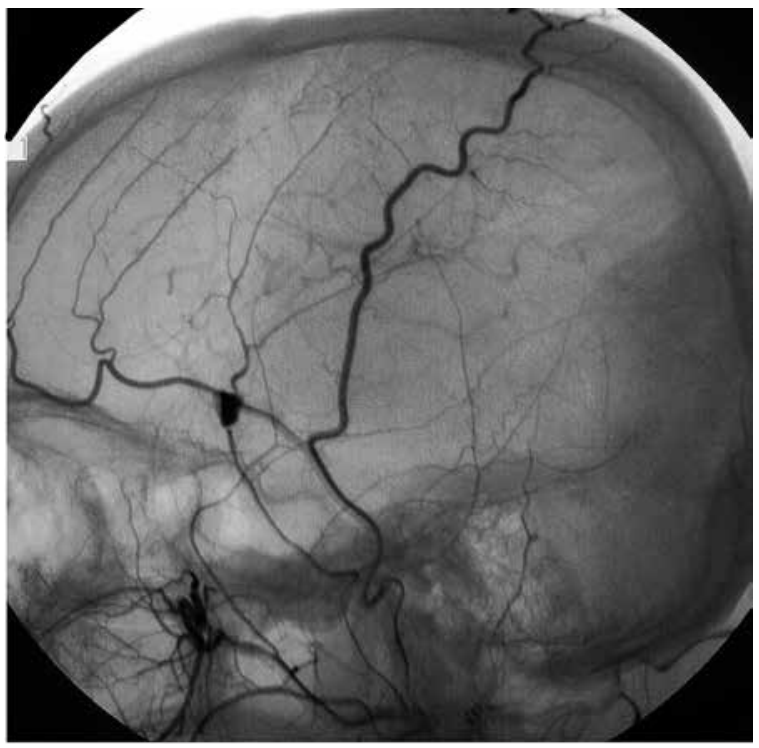

Figura 2 - Angiografia seletiva de artéria carótida externa direita. (A-B) incidência frontal; (C-D) incidência lateral demonstrando pseudoaneurisma da artéria meníngea média direita. 
A

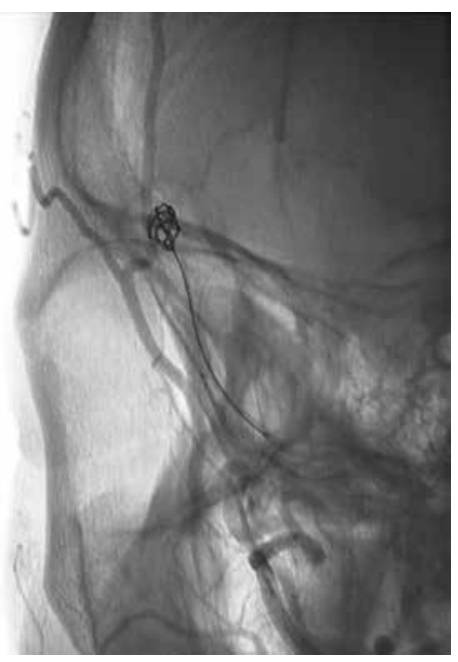

C

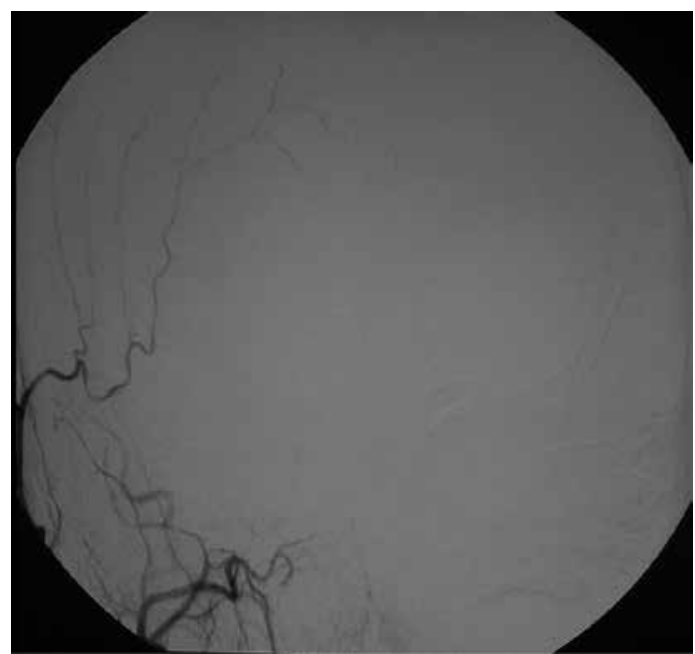

E

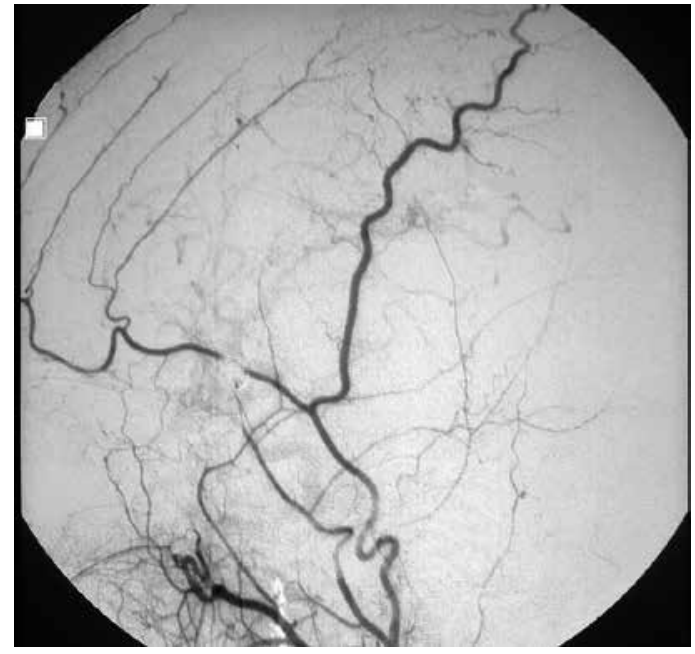

B

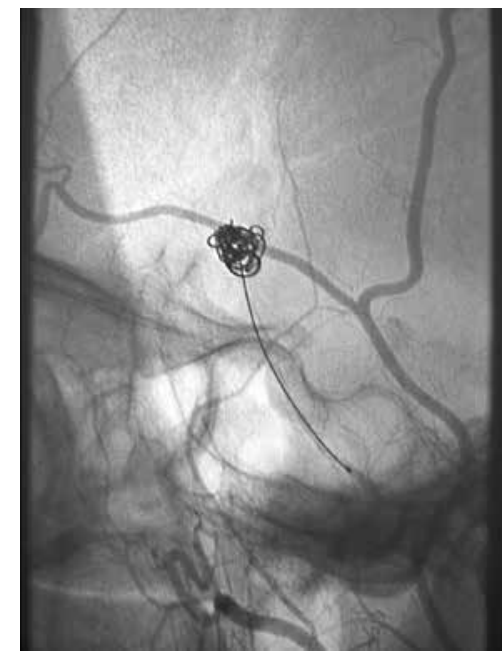

D

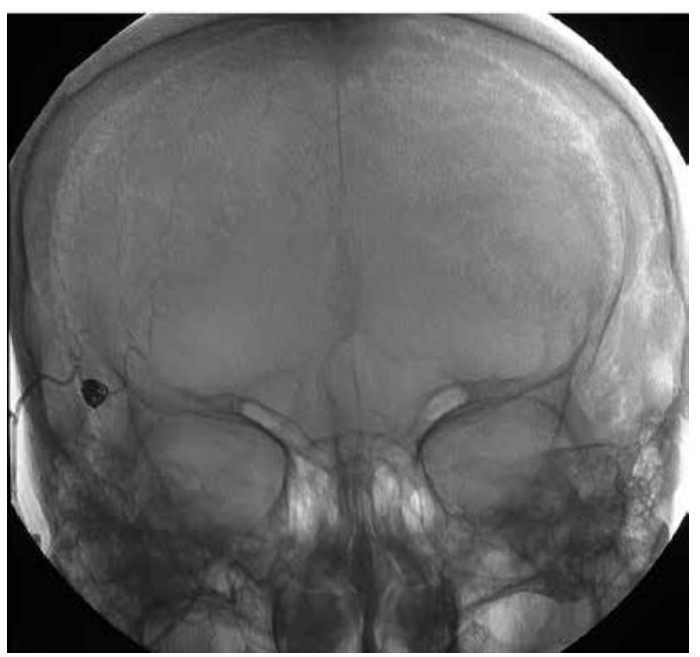

F

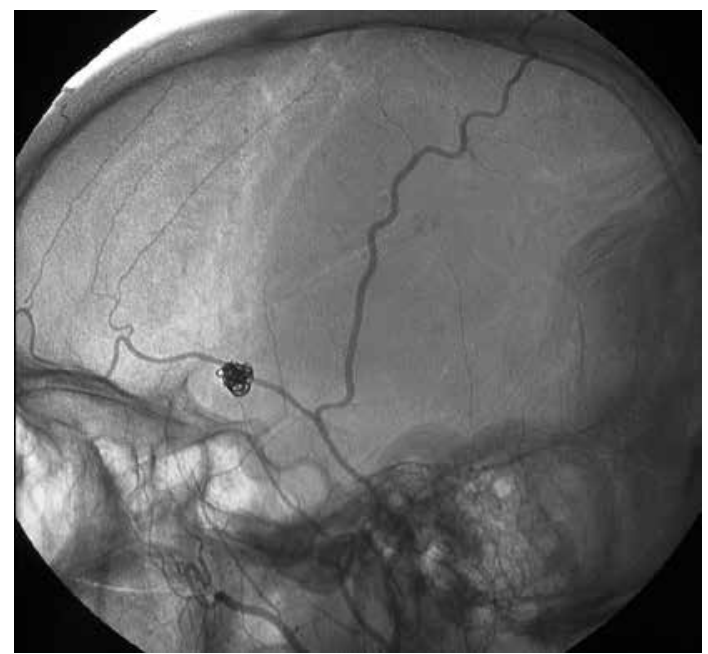

Figura 3 - (A-B) microcateterismo do pseudoaneurisma com preenchimento dele com coils; (C-F) angiografia seletiva da artéria carótida externa direita, após tratamento endovascular, demonstrando completa oclusão do aneurisma; (C-D) incidência frontal; (E-F) incidência lateral. 


\section{Discussão}

O primeiro caso de aneurisma traumático da AMM foi relatado por Schulze em $1957 .{ }^{3}$ A formação desses aneurismas está associada principalmente ao $\mathrm{TCE}^{2,5,6,8}$ e, nessas situações, as fraturas cranianas podem ser encontradas em até $92 \%$ dos casos. ${ }^{2,6,8} \mathrm{Na}$ maioria dos casos as fraturas cranianas são encontradas cruzando o trajeto da AMM, causando lesão da parede arterial com consequente formação do pseudoaneurisma. ${ }^{2-6,8,9}$ Cerca de $85 \%$ dos aneurismas traumáticos são encontrados na região temporal, ${ }^{6} 10 \%$ na occipital e $5 \%$ na região frontal. ${ }^{3}$

Há, ainda, relatos de hemorragia intracraniana por causa da ruptura não traumática de aneurismas da artéria meníngea média que podem estar associados principalmente a doença de Paget, malformações arteriovenosas, fístula arteriovenosa dural, hemangioma cavernoso do crânio, meningeoma, doença de Moyamoya, oclusão da artéria carótida interna ou da artéria cerebral posterior. ${ }^{7}$ É descrita também a formação de pseudoaneurisma de AMM devida a lesões iatrogênicas durante neurocirurgias eletivas. ${ }^{1}$

A maior incidência de aneurismas de AMM está entre os pacientes jovens e do gênero masculino, ${ }^{1,8}$ provavelmente por ser esse o grupo mais acometido por traumas cranianos. Aneurismas traumáticos da AMM estão associados a hematoma extradural em cerca de $61 \%$ dos casos. ${ }^{8}$ Podem, ainda, estar associados a hematomas: subdural, intraparenquimatoso ou intraventricular, ${ }^{2,6,7}$ numa frequência menor.

O diagnóstico dos aneurismas da AMM, na atualidade, é realizado por exames de angiotomografia, ${ }^{9}$ angiorressonância ou angiografia cerebral. ${ }^{1,4,6}$ São realizados quando há suspeita de lesão dessa artéria, habitualmente na presença de fratura temporal traumática. ${ }^{8,9}$ À angiografia, o aneurisma traumático da AMM está localizado perifericamente, distante do ponto de ramificação dessa artéria, e não possui colo. ${ }^{2,3,6}$ Caracteristicamente, seu fundo é irregular, o esvaziamento é demorado e os segmentos pré e pós- aneurismático não se opacificam ao mesmo tempo., 2,36

Embora seja o exame de maior sensibilidade para a detecção de aneurismas intracranianos, a AGC realizada logo após o trauma detecta aneurismas traumáticos em somente $54 \%$ dos casos. ${ }^{1}$ Assim, se houver forte suspeita da ocorrência de aneurismas traumáticos e a AGC inicial não demonstrar sua presença, uma nova arteriografia pode ser realizada duas semanas após. ${ }^{1}$ No presente caso, curiosamente, a angiografia cerebral foi realizada diante da suspeita de lesão da artéria carótida interna em sua porção petrosa, a qual não foi confirmada. A alteração constatada foi a presença de pseudoaneurisma da artéria meníngea média.
A história natural desse tipo de aneurisma não é bem conhecida. ${ }^{2,8}$ Sabe-se que pode evoluir com trombose espontânea, ${ }^{2}$ aumento de tamanho ou mesmo ruptura., ${ }^{2,8}$ Assim, o crescimento progressivo desses aneurismas pode ser demonstrado por angiografias seriadas. ${ }^{2} \mathrm{O}$ sangramento ativo da lesão da artéria meníngea média ocasiona a formação de hematoma extradural. ${ }^{6,8}$ Após intervalo lúcido variável, pode ocorrer rápida expansão do hematoma, seguida de deterioração neurológica súbita, ${ }^{8}$ conferindo elevada morbidade e mortalidade a essa condição. ${ }^{10}$ Desse modo, essas lesões devem ser diagnosticadas e tratadas precocemente. ${ }^{2,8}$

O tratamento dos pseudoaneurismas da AMM pode ser realizado por meio de craniotomia e coagulação da artéria portadora, ou por via endovascular, ${ }^{8,9}$ como no presente caso. Volumosos hematomas extradurais devem ser tratados por craniotomia, com esvaziamento do hematoma e coagulação da artéria meníngea média. ${ }^{1,5,6,8,10}$

\section{Conclusão}

A formação de pseudoaneurisma traumático da AMM constitui evento raro, sendo ainda pouco diagnosticado. Seu tratamento deve ser sempre indicado pelo risco de ruptura e formação de hematoma intracraniano. Esse tratamento pode ser realizado por via endovascular com segurança.

\section{Referências}

1. Ohba S, Kuroshima $\mathrm{Y}$, Mayanagi K, Inamasu J, Saito R, Nakamura $Y$, et al. Traumatic aneurysm of the supraclinoid internal carotid artery-case report. Neurol Med Chir (Tokyo). 2009;49(12):587-9.

2. Paiva WS, De Andrade AF, Amorim RL, Figueiredo EG, Teixeira MJ. Traumatic pseudoaneurysm of the middle meningeal artery causing an intracerebral hemorrhage. Case Report Med. 2010;2010:219572.

3. Shah Q, Friedman J, Mamourian A. Spontaneous resolution of traumatic pseudoaneurysm of the middle meningeal artery. AJNR Am J Neuroradiol. 2005;26(10):2530-2.

4. Oyama H, Nakamura S, Ueyama M, Ikeda A, Inoue T, Maeda $\mathrm{K}$, et al. Acute subdural hematoma originating from the lacerated intracranial internal carotid arteries - case report. Neurol Med Chir (Tokyo). 2006;46(2):84-7.

5. Kawaguchi T, Kawano T, Kaneko Y, Ooasa T, Ooigawa H, Ogasawara S. Traumatic lesions of the bilateral middle meningeal arteries - case report. Neurol Med Chir (Tokyo). 2002;42(5):221-3.

6. Nayil K, Ramzan A, Makhdoomi R, Wani A, Zargar J, Shaheen F. Incidental traumatic pseudoaneurysm of the middle meningeal artery: case report and literature review. Turk Neurosurg. 2012;22(2):239-41. 
7. Kobata H, Tanaka H, Tada Y, Nishihara K, Fujiwara A, Kuroiwa T. Intracerebral hematoma due to ruptured nontraumatic middle meningeal artery aneurysm - case report. Neurol Med Chir (Tokyo). 2001;41(12):611-4.

8. De Andrade AF, Figueiredo EG, Caldas JG, Paiva WS, De Amorim RL, Puglia $P$, et al. Intracranial vascular lesions associated with small epidural hematomas. Neurosurgery. 2008;62(2):416-20.

9. Jussen D, Wiener E, Vajkoczy P, Horn P. Traumatic middle meningeal artery pseudoaneurysms: diagnosis and endovascular treatment of two cases and review of the literature. Neuroradiology. 2012;54(10):1133-6.
10. Wang CH, Lee HC, Cho DY. Traumatic pseudoaneurysm of the middle meningeal artery: possible indicators for early diagnosis in the computed tomography era. Surg Neurol. 2007;68(6):676-81.

Endereço para correspondência

Guilherme Brasileiro de Aguiar

Disciplina de Neurocirurgia. Departamento de Cirurgia. Faculdade de Ciências Médicas da Santa Casa de Misericórdia de São Paulo

Rua Cesário Motta Jr., 112, Vila Buarque

01221-900 - São Paulo, SP, Brasil

Telefone: (55 11) 2176-7000

E-mail: guilhermebraguiar@yahoo.com.br 